\title{
Comparative performance of a modified change vector analysis in forest change detection
}

\author{
K. NACKAERTS*, K. VAESEN, B. MUYS and P. COPPIN \\ Geomatics and Forest Engineering Group, Department of Land Management, Vital \\ Decosterstraat 102, 3000 Leuven, Belgium; e-mail: \{kris.nackaerts, krist.vaesen, \\ bart.muys,pol.coppin\}@agr.kuleuven.ac.be
}

(Received 26 June 2001; in final form 21 August 2003)

\begin{abstract}
Sustainable forest management requires accurate and up-to-date information, which can nowadays be obtained using digital earth observation technology. This paper introduces a modified change vector analysis (mCVA) approach and conceptually contrasts it against traditional CVA. The results of a comparative study between this change detection algorithm and three other widely used change detection algorithms: standardized differencing, ratioing and selective principal component analysis are summarized. Landsat Thematic Mappper (TM) imagery and detailed change maps of a forested area in Northern Minnesota were used. Change indicators (vegetation indices) were grouped into three conceptually independent categories corresponding to soil, vegetation and moisture characteristics. Change periods of two, four and six years were considered. All change detection outputs were multidimensional and of a continuous nature, and could therefore be subjected to a supervised maximum likelihood algorithm using identical data training sets. Change extraction accuracies were determined by computing overall accuracy and Kappa coefficients of agreement against independent reference datasets. The mCVA outperformed the three other change detection methods in all cases, and we have shown that there is a clear advantage in running mCVA with three change indicator inputs where each input comes from a different change indicator category. Further validations with more detailed reference data are needed to improve this method and test its performance for other types of change events.
\end{abstract}

\section{Introduction}

Renewable natural resources such as forest ecosystems are continually changing. Change is defined as an alteration in the surface components of a vegetation cover (Milne 1988) or as a spectral/spatial movement of a vegetation entity over time (Lund 1983). The rate of change can be viewed as either dramatic and/or abrupt, as exemplified by clear felling, or as subtle and/or gradual, such as growth of standing volume. Some forest cover modifications, including deforestation for land-use conversion, are human induced while other modifications have natural origins, resulting from, for example, insect and disease epidemics. Change can be said to

*Corresponding author. 
have occurred when it can be shown that a vegetation cover entity has significantly different characteristics when viewed on at least two separate moments in time.

Monitoring techniques based on multispectral satellite-acquired data have demonstrated potential as a means to detect, identify and map changes in forest ecosystems (Coppin and Bauer 1994). This type of digital change detection has the advantage of (1) being repeatable; (2) facilitating the incorporation of biophysically relevant features from the infrared and microwave parts of the electromagnetic spectrum invisible for the human eye; and (3) requiring relatively low operational costs. Over the years, different change detection methods (using different preprocessing techniques, change indicators, change detection algorithms, classification procedures, etc.) have been proposed. A synoptic review of the wide range of change detection algorithms can be found at Coppin et al. (2004). They cite the standard Change Vector Analysis (CVA) as a change detection tool that characterizes movement in spectral space over time in terms of magnitude and direction.

The CVA approach defines a change vector as the difference vector between two vectors in an $n$-dimensional ( $n=$ number of change indicators) feature space, whereby these two vectors correspond to two observations of the same pixel at two different moments in time. The change vector's length represents the magnitude of the change event in the spectral feature space, while its direction corresponds to the type of change. While vector length is a continuous variable, vector direction has an angular nature requiring some special handling. One approach is to assign the change vectors to $2^{n}$ sectors (figure $1(a)$ ) according to the positive and negative shifts in spectral space for the $n$ change indicators used (Michalek et al. 1993, Virag and Colwell 1987). The thus defined sector codes are assumed to correspond to different types of change, offering qualitative (categorical) information in addition to the quantitative magnitude of the change event. Another method (Malila 1980) makes use of sector codes describing the directional grouping of the change vector's angles (figure 1(b)).

In both cases, the occurrence of change is determined through a simple thresholding operation on the magnitude parameter, and the type of change is defined according to the corresponding sector code.

An important drawback of this approach is the need for reference information to be able to interpret the change vectors. On the one hand, the identification of change events based on change vector magnitude is only possible when reference data are available to define the change vector magnitude threshold value. On the other hand, reference data are needed to identify change events based on change vector direction (Malila 1980). The training data are used to group change vector angles into meaningful change events such as an increase in biomass versus decrease in biomass. Consequently, traditional CVA remains to a certain extent data-driven (imagegoverned) and automation is possible only insofar as the multitemporal imagery to be analysed for change is spectrally fully normalized and consistent over time and space (one threshold and/or one training dataset remain valid for all imagery).

The CVA method, as described above, entails other disadvantages. As sector codes are categorical numbers and therefore unique and discrete, no pixel can belong to more than one sector, and no intermediate types of change can be identified. CVA thus precludes the implementation of statistical classification algorithms (e.g. maximum likelihood classification, isodata-clustering) to detect and identify change on a categorical, or even continuous, scale. Moreover, subjectively 
(a)

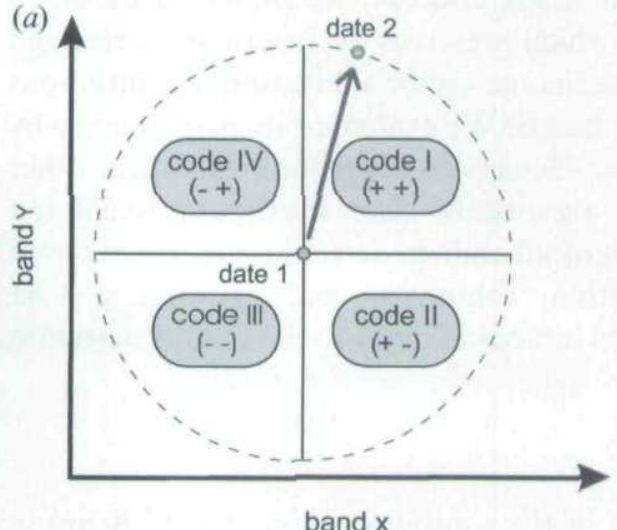

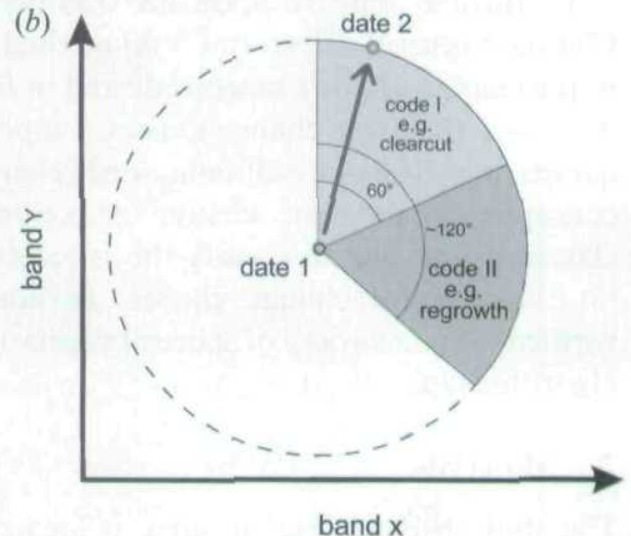

band $x$



Figure 1. Principles of change vector analysis with vector direction (a) categorized according to circle quadrants (Michalek et al. 1993); (b) categorized according to vector angle grouping (Malila 1980); (c) and magnitude both expressed as continuous values (mCVA).

setting magnitude thresholds for change-no change and/or defining change angle groupings (sectors) according to limited training datasets may induce considerable bias unless one is extremely knowledgeable about the area under study. When more than two input bands are involved (a minimum of $2 n$ training sites is needed for $n$ input bands), thresholding decisions and training procedures for sector definition (second method) quickly become much more complicated.

Allen and Kupfer (2000) proposed an expanded CVA approach by also using the information retained in the vector's spherical statistics in the change extraction process. However, they applied the sector coding approach for identifying the change event, and thus also maintained some of its inherent drawbacks. 
To further improve upon the traditional CVA concept, we present a modified Change Vector Analysis (mCVA) method, which preserves the information retained in the change vector's magnitude and in the change vector's direction as continuous data, and this for $n$ change indicator input bands. We evaluated its performance by comparing it as a self-contained change detection algorithm to three other conceptually different change detection algorithms. The maximum likelihood classifier was used to classify the outcomes of all change detection algorithms used into meaningful change classes. In addition, robustness and consistency were verified using a variety of spectral vegetation indices as input to the change detection algorithms.

\section{Materials}

The study site, $421 \mathrm{~km}^{2}$ in area, is located in the southwestern corner of Beltrami County in north central Minnesota (figure 2). The geomorphology of the area is northern glacial till plain. The landscape is nearly level to gently rolling with loamy soils that developed on the calcareous till. Generally these have a high water holding capacity and good drainage.

The local climate is characterized by wide extremes in temperature from summer to winter. The yearly precipitation ranges from 563 to $640 \mathrm{~mm}$. The area is characterized by a predominant, although not continuous, forest cover encompassing the intricate mixture of tree species and vegetation types that is typical of Northern Minnesota. Major tree species include aspen (Populus spp.); birch (Betula spp.); balsam fir (Abies balsamea); jack pine (Pinus banksiana); red pine (Pinus resinosa); white pine (Pinus strobus); black spruce (Picea mariana); white spruce

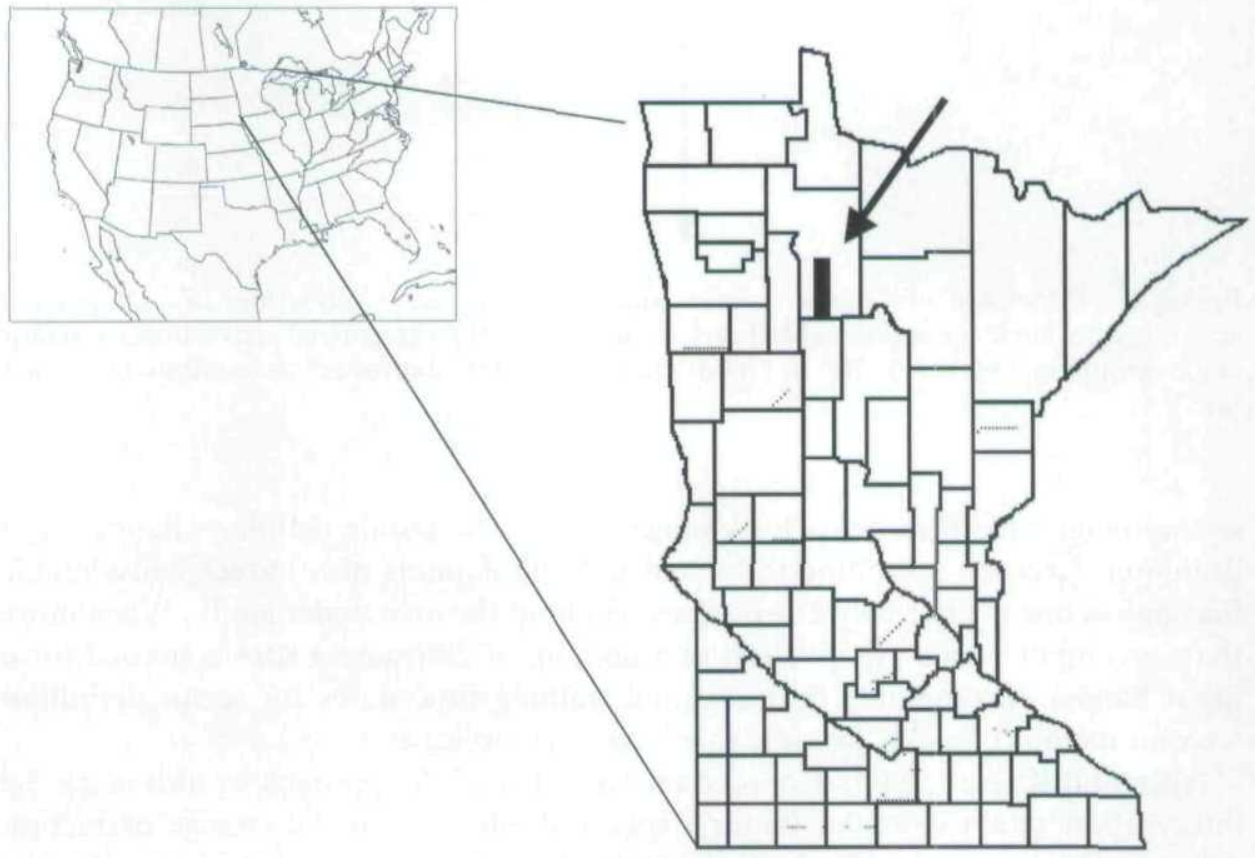

Figure 2. Location of the study area $(42000 \mathrm{ha})$. 
(Picea glauca); tamarack (Larix laricina); and northern hardwoods (Fraxinus nigra, Quercus rubra, Tilia spp., Acer spp., etc.).

The landscape is extremely fragmented, with an average forest stand area of about 3.4 ha. The forests in this part of Minnesota are complex, with an intricate mixture of different tree species, development stages and stand densities. Aspen/birch and jack pine are the dominant tree species and together account for about half of the stands. About $60 \%$ of all stands can be considered merchantable and $50 \%$ of the forest land area is well-stocked. There are about 1500 ha of over-mature forest, mostly aspen, with many of these stands showing symptoms of gradual aspen dieback due to Hypoxylon cancer (Hypoxylon mammatum). The forests have been under active management since at least the early 1980s.

Detailed digital forest maps with changes at the stand level over 2-, 4- and 6-year periods were available from a previous study (Coppin and Bauer 1995). The change classification was qualitative in nature with the following change categories: clearcut, clear-cut with natural regeneration, clearcut with plantation, flooding, highgrading/selective cut, complete vegetation removal, storm damage, early regeneration development, early plantation development, plantation establishment, and no change. Because the objective of this research was not the determination of the causal agent of a change event but the cross-evaluation of different change detection methodologies, the 10 change categories were regrouped into three major change types: net canopy loss (first six), net canopy gain (next three) and no change (last category). Table 1 summarizes the absolute (number of pixels) and relative (percentage of total area) occurrence of the change events for each of the three time intervals under consideration.

All development and validation of digital change detection methods was carried out using Landsat Thematic Mapper (TM) imagery covering the same area and the same time intervals $(1984,1986,1990)$, thus enabling an evaluation of the potential of the methods for mid-cycle forest inventory updating over 2-, 4- and 6-year

Table 1. Distribution of change events for 2-, 4- and 6-year intervals.

\begin{tabular}{|c|c|c|c|c|c|c|c|}
\hline & \multirow{2}{*}{$\begin{array}{c}\text { Change } \\
\text { class }\end{array}$} & \multicolumn{2}{|c|}{ 2-year period } & \multicolumn{2}{|c|}{ 4-year period } & \multicolumn{2}{|c|}{ 6-year period } \\
\hline & & $\begin{array}{l}\text { No. } \\
\text { pixels }\end{array}$ & $\begin{array}{l}\text { Area } \\
(\%)\end{array}$ & $\begin{array}{l}\text { No. } \\
\text { pixels }\end{array}$ & $\begin{array}{c}\text { Area } \\
(\%)\end{array}$ & $\begin{array}{l}\text { No. } \\
\text { pixels }\end{array}$ & $\begin{array}{c}\text { Area } \\
(\%)\end{array}$ \\
\hline Clearcut & NCL & 208 & 0.166 & 2187 & 1.742 & 2312 & 1.842 \\
\hline Clearcut with natural regeration & NCL & 1320 & 1.052 & 2014 & 1.605 & 3430 & 2.733 \\
\hline Clearcut with plantation & NCL & 986 & 0.786 & 3351 & 2.670 & 4424 & 3.525 \\
\hline flooding & NCL & 300 & 0.239 & 356 & 0.284 & 2506 & 1.997 \\
\hline Highgrading/selective cut & NCL & 148 & 0.118 & 833 & 0.664 & 1595 & 1.271 \\
\hline Complete vegetation removal & NCL & 41 & 0.033 & 78 & 0.062 & 105 & 0.084 \\
\hline Storm damage & NCL & 2036 & 1.622 & 36 & 0.029 & 496 & 0.395 \\
\hline Early regeneration development & NCG & 166 & 0.132 & 1646 & 1.311 & 415 & 0.331 \\
\hline Early plantation development & NCG & 5231 & 4.168 & 8650 & 6.892 & 9686 & 7.717 \\
\hline Plantation establishment & NCG & 171 & 0.136 & 212 & 0.169 & 309 & 0.246 \\
\hline No change & NC & 114909 & 91.549 & 406153 & 84.573 & 100238 & 79.861 \\
\hline NCL & & 5039 & 4.015 & 8855 & 7.055 & 14868 & 11.846 \\
\hline NCG & & 5568 & 4.436 & 10508 & 8.372 & 10410 & 8.294 \\
\hline NC & & 114909 & 91.549 & 106153 & 84.573 & 100238 & 79.861 \\
\hline
\end{tabular}

NCL, net canopy loss.

NCG, net canopy gain.

$\mathrm{NC}$, no change. 
periods. Acquisition dates were selected within a peak-green July-August time window for reasons of seasonal maturity and therefore phenological stability (Coppin and Bauer 1994).

\section{Methods}

An overview of the processing chain implemented in this research is given in figure 3 .

\subsection{Image preprocessing}

Preprocessing of the satellite imagery encompassed radiometric calibration to exoatmospheric reflectance, image registration, and image rectification. As terrain relief was nearly level to gently rolling, and in order to preserve the spectral characteristics of the imagery, a second order polynomial warp function combined with a nearest neighbourhood resampling technique were implemented during geocoding to the UTM-grid. The final RMS error was less than a quarter pixel, considered more than adequate for the purpose of this study. Finally, atmospheric normalization, masking of the non-forest lands, and interpretability enhancement via change indicator generation (see section 3.3) were applied.

\subsection{Change indicator or input band selection}

While it is commonly accepted that the six reflective TM bands fit in only three dimensions of spectral feature space (brightness, greenness, and wetness (Crist and Cicone 1984)), and that vegetation indices have the inherent capability of providing vegetation-related information not available in any single band (Wallace and Campbell 1989), no consensus can be found in the literature on which data transformations or indices best represent biophysical features in the context of green vegetation monitoring. Moreover, other factors such as the characteristics of the underlying soil, viewing and illumination geometry may play a decisive role (Huete and Jackson 1985).

Among all possible change indicators (single TM bands, vegetation indices, and linear transformations) with their widely differing degrees of correlation, three major groups may be differentiated, each more directly linked to a specific biophysical property of the forest ecosystem. A first group combines those indicators that were proven to contain information related to soil characteristics, a second combines those that relate to vegetation properties, and a third group combines those that were shown to correlate with moisture status. It was therefore decided to investigate the most commonly used indicators within each of the three groups: the single TM3 band, 'red' and the Tasseled Cap brightness 'bright' (Kauth and Thomas 1976) for the first group; the single TM4 band 'vnir', the tasseled-cap greenness 'green', the Normalized Difference Vegetation Index 'ndvi', and the SoilAdjusted Vegetation Index 'savi' for the second group; and the single TM5 band 'mir', the tasseled-cap wetness 'wet', and the TM5/TM2 ratio 'mg' for the third group.

The Tasseled Cap or Kauth-Thomas transformation orthogonally transforms raw data into a new coordinate system whereby each axis is independent (orthogonal) and corresponds to a distinct biophysical characteristic. It has been shown repeatedly that the second axis, denoted as 'greenness', contains mainly vegetation-specific information, while the first one (referred to as 'brightness') is more correlated to differences in soil reflectance. A third axis is referred to as 


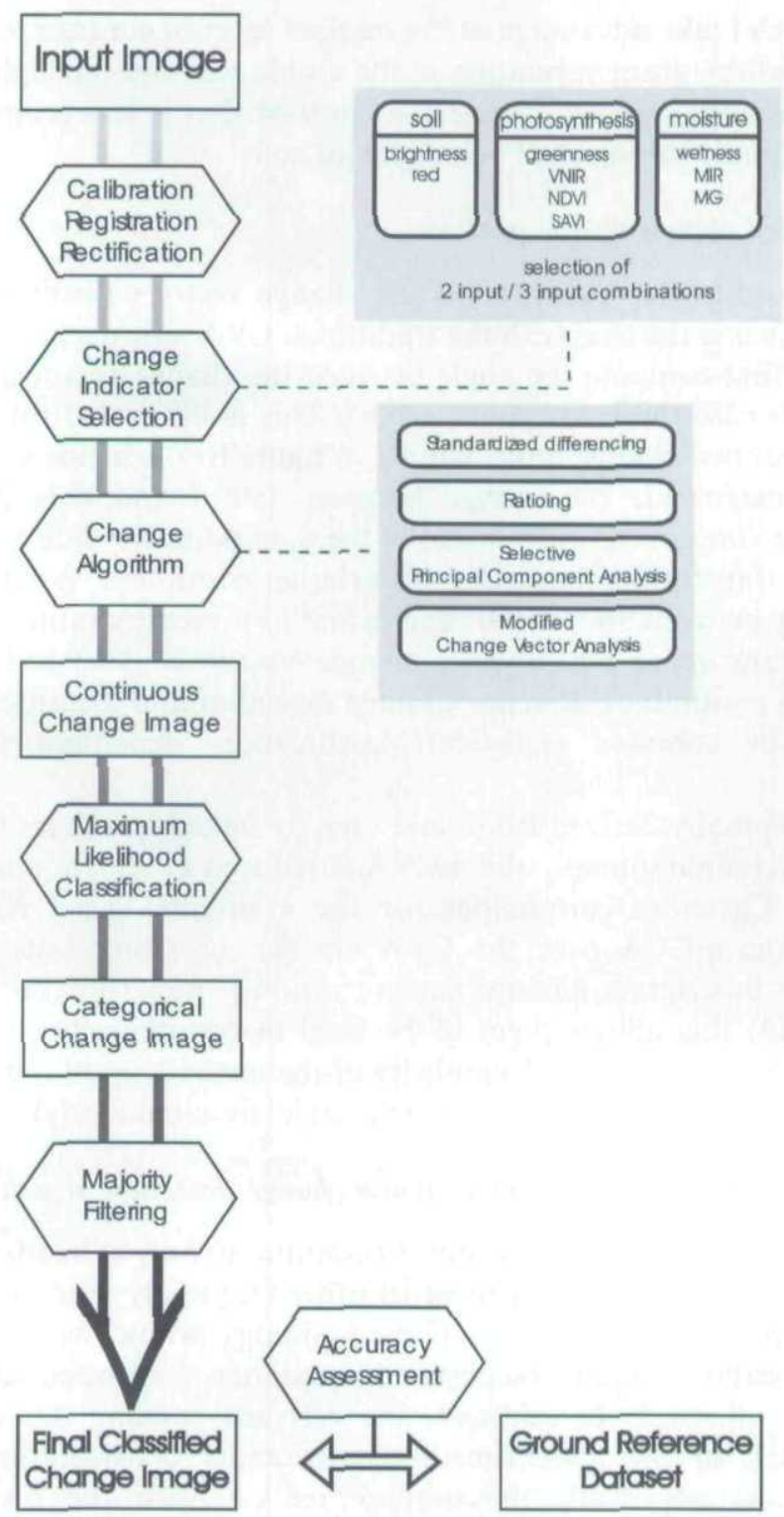

Figure 3. Overview of the overall change detection methodology.

'wetness', which is generally linked to moisture status. Kauth-Thomas coefficients for exo-atmospheric reflectance factors were taken from Crist and Cicone (1984), NDVI (Rouse et al. 1973) was computed as shown in equation (1):

$$
\mathrm{NDVI}=(\mathrm{VNIR}-\mathrm{RED}) /(\mathrm{VNIR}+\mathrm{RED})
$$

To correct for soil influences, Huete (1988) modified the NDVI into the SAVI as given by equation (2):

$$
\mathrm{SAVI}=((\mathrm{VNIR}-\mathrm{RED}) \times(1+L)) /(\mathrm{VNIR}+\mathrm{RED}+L)
$$

with $L=0.5$ 
NDVI and SAVI take advantage of the marked spectral contrast between the low reflectance of healthy green vegetation in the visible red, and the high reflectance in the very-near-infrared spectral channels, a contrast that is less prominent where it concerns for example the spectral behaviour of soils.

\subsection{The modified change vector analysis}

To obtain a quantitative descriptor of the change vector's direction instead of a qualitative one (being the case with the traditional CVA approaches as illustrated in figure $1(a)$ ), we first compute the angle between the change vector and a reference direction, in this case the horizontal (x-axis). This is illustrated for a simple twodimensional case (two change input bands) in figure $1(c)$. Change vector angle and change vector magnitude for change between date 1 and date 2 can thus be considered polar coordinates with origin in the data point for date 1. Second, polar coordinates are transformed back into a Cartesian coordinate system to overcome the discontinuity between $360^{\circ}$ and $0^{\circ}$ angles and to preserve continuity. The overall result is a feature space where each change vector is described by Cartesian coordinates in a continuous domain. Change detection and identification can now be performed by common statistical classification algorithms (supervised or unsupervised).

Though conceptually derived from, and directly linked to the traditional change vector analysis, computationally the mCVA is reduced to simple multidimensional differencing of Cartesian coordinates for the $n$ original input bands. Obvious advantages of the mCVA over the CVA are (1) the continuous nature of the resulting change descriptors (change categorization is now fully in the continuous data domain), (2) this allows them to be used in common change classification algorithms, (3) the computational simplicity of the method, and (4) its feature space multidimensionality ( $n$ input bands can be treated simultaneously).

\subsection{Cross-referencing against other common change detection algorithms}

To enable a comparison of mCVA results ( $n$ continuous output bands resulting from $n$ input change indicators) against those of other frequently used change detection algorithms so that only the choice of methodology would have an impact, an identical classification routine (supervised maximum likelihood algorithm) was implemented in all cases. In addition, the relevant training datasets were also identical for each of the three time intervals under consideration. From that perspective it was impossible to compare mCVA with the traditional CVA independently, as the former produces $n$ output layers $(n=$ the number of input bands) that have a continuous character and thus lend themselves to statistical classification routines, while the latter results in an operator-controlled straightforward characterization of the change events, without intervention of any statistical routines.

Because of the inherent differences with mCVA, and with each other, image differencing (Singh 1989), image ratioing (Jensen 1981), and selective principal component analysis (Coppin and Bauer 1994) were selected for cross-referencing. Image differencing is probably the most widely applied change detection algorithm. It involves pixel-level subtraction of one date of imagery from a second date after the latter has been precisely registered and normalized to the first. By standardizing the difference (difference divided by the sum) the occurrence of identical change values, depicting different change events, is minimized (Coppin and Bauer 1996). 
The second algorithm, image ratioing, is one of the computationally simplest and quickest change detection methods. Data are ratioed on a pixel-by-pixel basis. A pixel that has not changed receives a ratio value of one. Areas of change will have values either higher or lower than one, depending on the type of change. Change indicator imagery from two separate dates can also be statistically transformed into a 2-dimensional spectral space, and this for each change indicator separately. The most frequently applied transformation technique is principal component analysis (PCA). PCA transforms the feature space coordinate system into a new equallydimensioned Euclidean coordinate system by means of a linear transformation in such a way that maximal variability is located along the first new axis (PC1). To create statistical independence, the second axis (PC2) is constructed orthogonally to the first. For change detection, only two inputs of a single change indicator, however acquired at different points in time, are processed and the algorithm is referred to as selective PCA. If the change area is small proportional to the total study area, the first component will contain information on the intra-image variability and the second on the inter-image variability, and thus on the temporal change (Chavez and Kwarteng 1989). Because of this, only PC2 can be considered a change descriptor and therefore it has been used in this study. To develop the principal component data transformation, the eigenvalues were calculated from the correlation matrix because both Fung and LeDrew (1987) and Eastman and Fulk (1993) demonstrated this approach is superior to a covariance-matrix-based method.

As is evident from the previous paragraphs, mCVA necessitates at least two change indicators as input. Because of the categorization of the change indicators into three biophysically meaningful groups, and in order to explore the robustness of the performance of the mCVA relative to the other change detection algorithms, it was decided to run all four change detection algorithms on all different combinations of pairs of indicators from the three groups (26 possible pairs). In other words, a supervised maximum-likelihood classifier was run on all results of mCVA output (two bands), and on all paired results of the three other change detection algorithms implemented for the same two indicators individually (two bands). To test whether additional and/or complementary spectral information (contained in a third indicator) would give a superior change detection outcome, three-input-band combinations also were implemented in a similar fashion (i.e. all triple combinations with each indicator pertaining to a different group). Finally, all classification results were subjected to a $3 \times 3$ majority filter to diminish the salt-andpepper appearance common for pixel-based digital classification results, and to better approximate the forest stand concept.

\subsection{Method validation}

Identical time-interval-specific reference datasets were used to validate the change detection results of all methods. Furthermore, these reference datasets were also totally independent from the classification training data in order to avoid any possible biases. Overall accuracies (total percentages correct) and Kappa coefficients of agreement (Congalton and Mead 1983) were computed against these standard reference datasets for each time interval, each change detection algorithm, and each combination of twin and triple change indicators. Kappa coefficients of agreement measure the observed agreement between classified and reference data as reported by the diagonal entries in the confusion matrix minus the agreement that might be contributed solely by a chance matching of the two datasets. Finally, the Kappa 
values were subjected to statistical analysis, involving comparison of the Kappa distributions (over all change indicator combinations) for paired change detection algorithms. Statistical significance of the observed differences between algorithm combinations was verified by implementing paired and independent sample tests.

\section{Results and discussion}

Figures 4 and 5 summarize, respectively for the two-band-input and for the threeband-input cases, the results of the computations of the Kappa coefficients of agreement (the darker the grey scale in the visualization, the lower the Kappa value and thus the accuracy). Because of the design of our validation procedure (standard statistical classification routine, standard training data, identical number of inputs), differences in Kappa values can be attributed solely to differences in choice of change detection algorithm for each combination of change indicators and for each time interval.

From the paired sample tests between twin Kappa distributions of mCVA and the other change detection algorithms, and confirmed, insofar as possible, by visual

\section{2 year period}

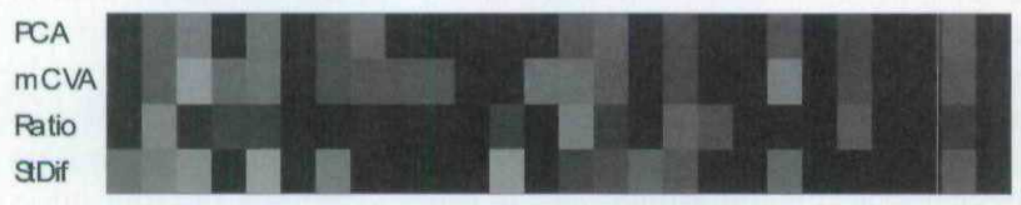

\section{4 year period}

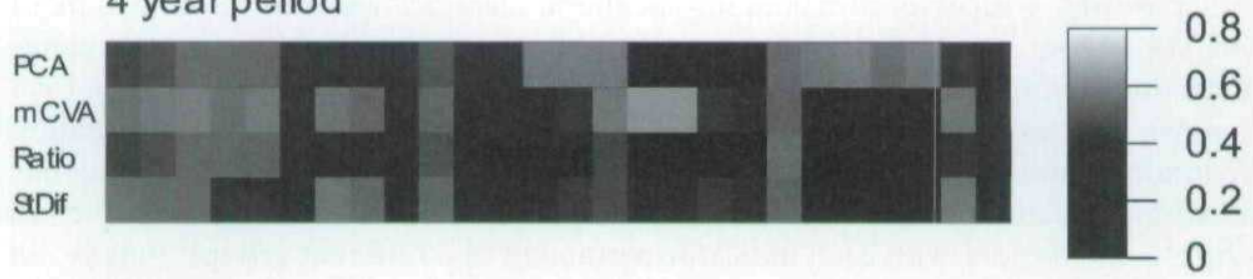

\section{6 year period}

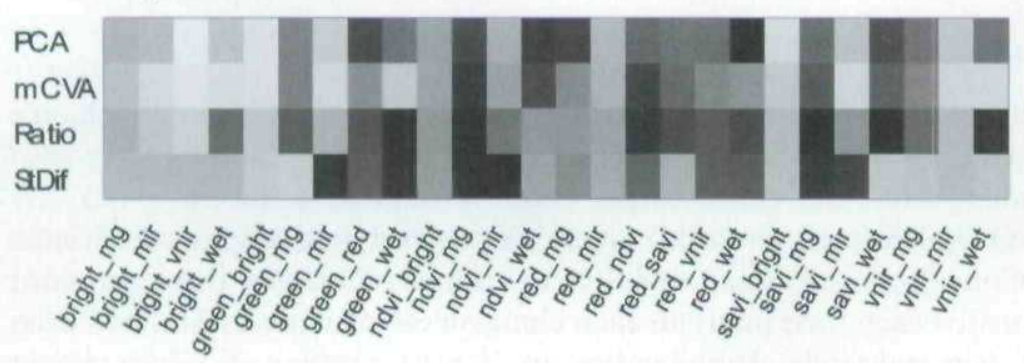

Figure 4. Summary of Kappa coefficients of agreement for two input realizations where bright $=$ Kauth - Thomas $(\mathrm{KT})$ brightness; $\mathrm{mg}=\mathrm{TM} 5 / \mathrm{TM} 2$; $\operatorname{vnir}=\mathrm{TM} 4$; wet=KT wetness; green $=\mathrm{KT}$ greenness; $\mathrm{red}=\mathrm{TM} 3$; ndvi $=\mathrm{NDVI} ; \mathrm{mir}=\mathrm{TM} 5$; savi=SAVI. 


\section{2 yearperiod}

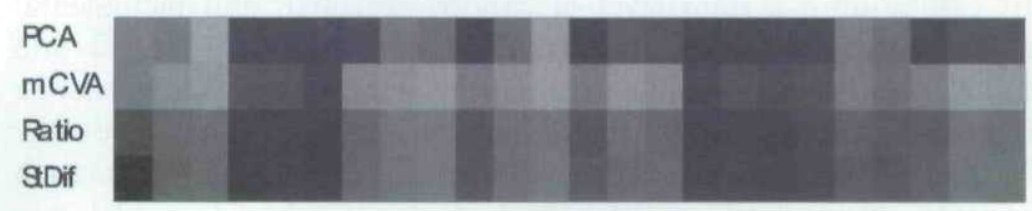

\section{4 year period}

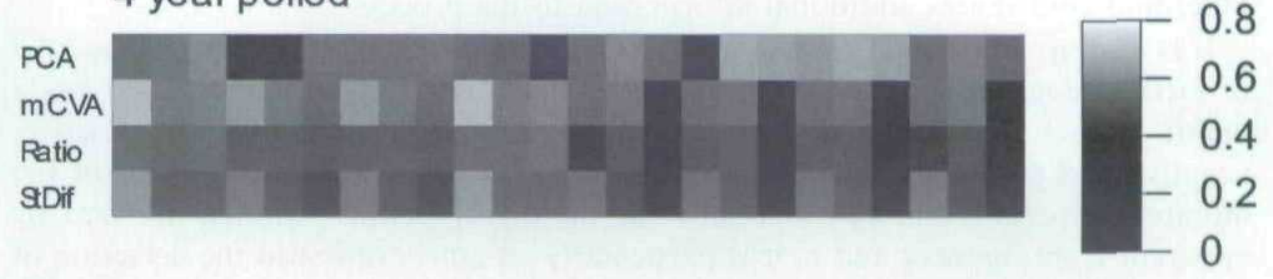

Kappa

\section{6 year period}
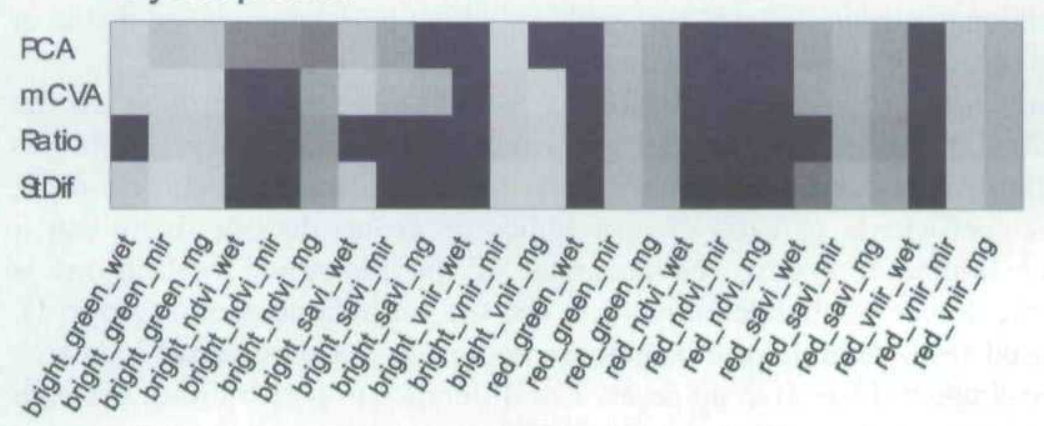

Figure 5. Summary of Kappa coefficients of agreement for three input realizations where bright $=\mathrm{KT}$ brightness; $\mathrm{mg}=\mathrm{TM} 5 / \mathrm{TM} 2$; $\mathrm{vnir}=\mathrm{TM} 4$; wet $=\mathrm{KT}$ wetness; green $=\mathrm{KT}$ greenness; red=TM3; ndvi $=$ NDVI; $\operatorname{mir}=$ TM5; savi $=$ SAVI.

analysis of figures 4 and 5, we conclude that, overall, our mCVA performs significantly better than the other algorithms. Kappa coefficients are in most cases significantly higher (at $\alpha=0.05$ ) than those resulting from the other change detection algorithms, irrespective of the time interval and the number of input bands. Two cases where the difference is not statistically significant, however, are those between mCVA and standardized differencing and between mCVA and PCA, respectively, for the 2-year and 4-year change detection intervals, both times for the two-input scenario.

Figures 4 and 5 (confirmed by independent sample tests) equally illustrate that mCVA is less sensitive to the choice of change indicator combination, the grey scale of the mCVA rows being lighter and more uniform in all six cases (two- and threeinput, 2-, 4- and 6-year periods). It is also evident that the addition of a third change indicator results in improved accuracies, except for the three-input case for the 6year period. Here it is evident that change detection is not improved for some combinations of change indicators. This may be explained by the fact that, the 
longer the time interval after the actual change event, the more negative change events also start exhibiting a certain level of canopy regrowth and incremental overshadowing, which first and foremost seems to negatively influence the soil- and moisture-linked change indicators. We are not able to ascertain, however, which one of these two indicator groups has the major impact. On the whole, one may conclude that the input in the change detection algorithm of one indicator from each of the three indicator groups (soil-, vegetation- and moisture-based) results in a significantly better change detection outcome as compared to twin-inputs only. This confirms the usefulness of the categorization of the change indicators into three groups based on biophysical reality, as each of the groups is complementary to the other and contributes additional information to the process.

It is also apparent that, for the given change categories (kept simple for the sake of methodological comparison), the length of the change detection interval has a major impact. For the two-input cases, overall change detection for positive, negative and stationary canopy evolution improves with increasing length of the monitoring period, and this especially for the 6-year period. Though not directly apparent from figures 4 and 5, it is particularly an amelioration in the detection of positive change events (canopy growth) that contributes to this global improvement. The same cannot be said for the three-input cases (see above). It is, however, also true that the total number of change events available for validation increased with the length of the monitoring interval (see table 1), which may have played a role in the representativeness of the Kappa coefficients.

The selection of individual change indicators from the respective groups, on the other hand, does indeed affect the change detection accuracy where mCVA is concerned. Although independent sample tests $(\alpha=0.05)$ between the two-input mCVA Kappa coefficients of each change indicator group do not single out a superior change indicator group, the accuracy of the individual realizations is dependent on the choice of change indicators. For the soil related group (group 1), the sample paired tests demonstrate brightness being the more powerful indicator (better than the single red band) in all cases. The difference in performance between the indicators of group two (group related to vegetative processes and structures) is however less distinct. All group-two indicators perform equally well, except for NDVI, which in all cases gives significantly lower Kappa coefficients. Finally, the single MIR band (TM5) appears to be the change indicator with the highest changerelevant information content in the moisture related group (group three), and wetness the least relevant indicator.

\section{Conclusions}

The modified Change Vector Analysis algorithm presented in this paper has proven to be a promising tool for forest cover change detection. Major advantages lie in its computational efficiency and in its information extraction capability. With respect to the first, the mCVA is capable of processing any number of change indicator bands simultaneously delivering outputs that have a continuous nature and therefore can be subjected to statistical change feature extraction. Moreover, training data are required only in the feature extraction phase, which can be based on any possible classification algorithm, e.g. supervised or unsupervised classifiers, neural networks. This is in contrast to traditional CVA which necessitates reference data for change-no change thresholding in the magnitude domain, and again for angle grouping when Malila's method is implemented. As to the second advantage, 
mCVA takes into account not only the magnitude of the changes detected, but equally so their directions, however preserved in a continuous domain. The other change detection algorithms against whom mCVA was cross-referenced deliver magnitude outputs in the absolute domain only (differences, ratios, and values of the second PC of a selective PCA, and this for a single change indicator at a time, at two points of the change detection time-scale), basically neglecting the change direction, and thus change type concept.

From the validation phase, one may deduce that for forest cover monitoring, the three-input mCVA performed better than the two-input mCVA, and this especially so for the shorter monitoring intervals ( 2 and 4 years). The combined input of one change indicator of each of the three indicator groups (soil-, vegetation-, and moisture-based - biophysically 'less' correlated) into the mCVA procedure certainly contributed to this outcome. Though we found relationships between the choice of individual change indicators from within the groups and change detection algorithm performance, the coarseness of the change classes (canopy depletion, canopy increment and no change) does not allow for final conclusions in this respect. To confirm these trends, one would need reference datasets with enough (temporally and spatially) occurrences of the specific change events. Such datasets are extremely hard to come by. For this, a standardized methodology should be developed to identify and quantify various change events more accurately. Though not verifiable, historical timber logging information could be used and converted into semiquantitative change classes of interest. In combination with the analysis of aerial imagery for several selected regions of interest, this could provide a more standardized dataset, especially when combining information from different counties. However, a better approach to assess the magnitude of change is to use a quantitative in situ measurable parameter. A possible parameter for this is the leaf area index (LAI) or the one-sided total leaf area per unit ground area which can be measured via specialized instruments. The main drawback of this is that no historical records of LAI exist in an operational forest management setting. An experimental setup would therefore be needed whereby various types of change events are induced via girdling and thinning operations to simulate events relevant for forest management. In this way, maximal variability in change events could be reached over a minimum area.

\section{References}

Allen, T.R. and KuPFER, J.A., 2000, Application of spherical statistics to change vector analysis of Landsat data: Southern Appalachian spruce-fir forests. Remote Sensing of Environment, 74, pp. 482-493.

ChAveZ, P.S.J. and KWARTENG, A.Y., 1989, Extracting spectral contrast in Landsat Thematic Mapper image data using selective principal component analysis. Photogrammetric Engineering and Remote Sensing, 55, pp. 339-348.

Congalton, R.G. and Mead, R.A., 1983, A quantitative method to test for consistency and correctness in photointerpretation. Photogrammetric Engineering and Remote Sensing, 49, pp. 69-74.

COPPIN, P.R. and BAUER, M.E., 1994, Processing of multitemporal Landsat TM imagery to optimize extraction of forest cover change features. IEEE Transactions on Geoscience and Remote Sensing, pp. 918-927.

COPPIN, P.R. and BAUER, M.E., 1995, The potential contribution of pixel-based canopy change information to stand-based forest management in the northern US. Journal of Environmental Management, 44, pp. 69-82. 
Coppin, P.R. and BAuer, M.E., 1996, Digital change detection in forest ecosystems with remote sensing imagery. Remote Sensing Reviews, 13, pp. 207-234.

Coppin, P.R., Lambin, E., Jonckheere, I., Nackaerts, K. and Muys, B., 2004, Digital change detection methods in ecosystem monitoring: a review. International Journal of Remote Sensing, 25, pp. 1565-1596.

Crist, E.P. and Cicone, R.C., 1984, Application of the Tasseled Cap concept to simulated Thematic Mapper data. Photogrammetric Engineering and Remote Sensing, 50, pp. 343-352.

EASTMAN, J.R. and FULK, M., 1993, Long sequence time-series evaluation using standardized principal components. Photogrammetric Engineering and Remote Sensing, 59, pp. 991996.

FunG, T. and LeDrew E., 1987, Application of principal components analysis to change detection. Photogrammetric Engineering and Remote Sensing, 53, pp. 1649-1658.

HuEtE, A.R., 1988, A soil-adjusted vegetation index (SAVI). Remote Sensing of Environment, 25, pp. 295-309.

HUETE, A.R. and JACKSON, R.D., 1985, Spectral response of a plant canopy with different soil backgrounds. Remote Sensing of Environment, 17, pp. 37-53.

JENSEN, J.R., 1981, Urban change detection mapping using Landsat digital data. The American Cartographer, 8, pp. 127-147.

Kauth, R.J. and Thomas, G.S., 1976, The Tasseled-Cap - a graphic description of the spectral-temporal development of agricultural crops as seen by Landsat. Proceedings of the 2nd Annual Symposium on Machine Processing of Remotely Sensed Data, Purdue University, West Lafayette, IN, USA, 21 June-1 July 1976 (West Lafayette, IN: Purdue University), pp. 41-51.

Lund, H.G., 1983, Change: now you see it-now you don't. Proceedings of the 19th International Symposium on Renewable Resource Inventories for Monitoring Changes and Trends, edited by J.F. Bell and T. Atterbury, College of Forestry, Oregon State University, Corvallis, OR, USA, 15-19 August 1983 (Corvallis, OR: Oregon State University), pp. 211-213.

MALILA, W.A., 1980, Change Vector Analysis: an approach for detecting forest changes with Landsat. Proceedings of the 6th Annual Symposium on Machine Processing of Remotely Sensed Data, Purdue University, West Lafayette, IN, USA, 3-6 June 1980 (West Lafayette, IN: Purdue University), pp. 326-336.

Michalek, J.L., WAgNer, T.W., Luczkovich, J.J. and Stoffle, R.W., 1993, Multispectral change vector analysis for monitoring coastal marine environments. Photogrammetric Engineering and Remote Sensing, 59, pp. 381-384.

MiLNE, A.K., 1988, Change direction analysis using Landsat imagery: a review of methodology. Proceedings of the 8th IGARSS Symposium on Remote Sensing: Moving towards the 21st Century, University of Edinburgh, Edinburgh, Scotland, 12-16 September 1988 (Edinburgh: University of Edinburgh), pp. 541-544.

Rouse, J.W. JR., HaAs, R.H., Schell, J.A. and Deering, D.W., 1973, Monitoring vegetation systems in the Great Plains with ERTS. Proceedings of the 3rd Earth Resources Technology Satellite-1 Symposium, Washington, DC, USA, 10-15 December 1973 (Washington, DC: NASA), vol. 1, pp. 309-317.

SingH, A., 1989, Digital change detection techniques using remotely-sensed data. International Journal of Remote Sensing, 10, pp. 989-1003.

VIRAG, L.A. and Colwell, J.E., 1987, An improved procedure of change in Thematic Mapper image-pairs. Proceedings of the 21st International Symposium on Remote Sensing of Environment, Ann Arbor, MI, USA, 26-30 October 1987 (Ann Arbor, MI: Environmental Research Institute of Michigan), pp. 1101-1110.

Wallace, J.F. and CAmpBell, H., 1989, Analysis of remotely sensed data. In Remote Sensing of Biosphere Functioning, R.J. Hobbs and H.A. Mooney (Eds), pp. 297-304. 
Copyright of International Journal of Remote Sensing is the property of Taylor \& Francis Ltd and its content may not be copied or emailed to multiple sites or posted to a listserv without the copyright holder's express written permission. However, users may print, download, or email articles for individual use. 